\title{
Optimization of a perfect absorber multilayer structure by genetic algorithms
}

\author{
Roberto Li Voti@
}

\begin{abstract}
Background: An increasing interest has been recently grown in the development of nearly perfect absorber materials for solar energy collectors and more in general for all the thermophotovoltaic applications.

Methods: Wide angle and broadband perfect absorbers with compact multilayer structures made of a sequence of ITO and TiN layers are here studied to develop new devices for solar thermal energy harvesting. Genetic Algorithms are introduced for searching the optimal thicknesses of the layers so to design a perfect broadband absorber in the visible range, for a wide range of angles of incidence from $0^{\circ}$ to $50^{\circ}$, and for both polarizations.

Results: Genetic Algorithms allow to design several optimized structures with 6, 8, and 10 layers reaching a very high average absorbance of $97 \%, 99 \%$ and $99.5 \%$ respectively together with a low hemispherical total emissivity (<20\%) from $200{ }^{\circ} \mathrm{C}$ till $400{ }^{\circ} \mathrm{C}$.

Conclusions: The proposed multilayer structures use materials with high thermal stability, and high melting temperature, can be fabricated with simple thin film deposition techniques, appearing to have very promising applications in solar thermal energy harvesting.
\end{abstract}

Keywords: Optical materials and properties, Perfect absorber, Multilayer structure, Thermophotovoltaic, Solar energy collectors

\section{Background}

During last decade a huge interest has been grown in the development of nearly perfect absorber materials for applications as solar energy collectors, and, more in general, for all the thermophotovoltaic applications (TPV), so to increase the absorbed power for energy harvesting, storage and conversion as well as for the reprocessing of wasted heat in industrial processes $[1,2]$.

The use of carbon nanotube technology improved a lot the performance of the absorbing materials. In 2009 nanomaterials based on vertically aligned single-walled carbon nanotubes shown an absorbance of about $98 \%$ of the incoming light in a wide spectral range UV-VIS-NIR [3]. But despite recent advances in the development of carbon nanotubes $(\mathrm{CNT})$ purity assessment tools, the macroscale assessment of the overall surface qualities of commercial $\mathrm{CNT}$ materials remains a great challenge,

Correspondence: roberto.livoti@uniroma1.i

Dipartimento di Scienze di Base ed Applicato per I'Ingegneria, Sapienza Università di Roma, Via A. Scarpa 16, 00161 Rome, Italy bringing negative impacts on the reliable and consistent nano-manufacturing of CNT products $[4,5]$.

A new concept of perfect absorber is now based on metamaterials where the enhancement of the absorption can be obtained thanks to the excitation of the surface plasmon resonance for example in gold and silver [6-13]. This can be achieved with structured metallic surfaces [14], microcavities [15], subwavelength hole arrays and opals $[16,17]$. Alternatively structured phase change materials [18-23] and chiral metamaterials [24-27] have been recently used so to obtain an active switching of the absorption properties thanks to the metal-insulator transition in one case, or to intrinsic/extrinsic dichroism in the other case. However, the realisation of broadband absorber metamaterials requires long and expensive procedures with multiple steps of film deposition, photoresist coating, etching and photoresist removing [28].

It should be underlined that the design and realisation of perfect absorbers with multilayers can be more technically and economically convenient [29]. The multilayer structure can be easily theoretically tested by numerical 
simulations, and optimized so to obtain broadband absorbers for a wide range of angles of incidence by using several standard search methods (Genetic Algorithms [30], Neural Network [31-33], Singular Value Decomposition [34-36], Steepest Descent Methods [37, 38], etc....). In addition the multilayered opaque structures are also easy to be characterized by using many different diagnostic techniques: photothermal, photoacoustic, photopyroelectric, and thermographic techniques [39-45].

Concerning the materials, an increasing interest is for the inorganic ceramic materials such as semiconductorbased oxides and transition-metal nitrides (TiN) which represent the alternative plasmonic materials in the visible frequencies [46-50], with a good thermal stability $[51,52]$.

Other common materials for optical electronic device applications and solar cells are the transparent conducting oxides (i.e. ITO) which can support surface plasmon polariton excitations $[53,54]$.

In this paper we study and optimize a multilayer structure based on a stack of ITO/TiN layers deposited onto a silver thick layer so to design the coating of a solar thermal collector. The idea of such a structure has been recently proposed in Ref. [49]. The authors designed an ITO/TiN multilayer finding an average absorbance of $90 \%$ in the visible for a wide range of incidence angle with 7 layers but without studying the infrared emissivity. Our purpose is to optimize this configuration by introducing a silica top layer in order to reach a higher average absorbance and by analysing the low thermal emissivity properties of the structure at different temperatures. We performed numerical simulations by changing the number of layers, and the layer thicknesses, and we applied the Genetic Algorithms to find the optimal thicknesses of the multilayer structure.

\section{Methods}

In this section we discuss the approach to design a ITO/ TiN multilayered structure to be an efficient coating for solar thermal collectors. The structure will be designed so to exhibit the highest average absorbance in the spectral range from $400 \mathrm{~nm}$ to $750 \mathrm{~nm}$ (visible window) for a wide range of incidence angles from $0^{\circ}$ to $50^{\circ}$, but also satisfying the requirement on a low thermal emissivity $(<20 \%)$ in the infrared, so to minimize the radiative losses of the solar collector.

The concept of multiband perfect absorbers by using metallo/dielectric multilayers has been recently introduced [55, 56]. It is known that 1D Photonic Band Gap structures can be tailored for specific photonic devices to exhibit exceptional properties, for example to obtain the transparency in metals [57-60], as well as to achieve the total absorbance [55] in a wide range of wavelengths. Regarding the perfect absorbance, metallo/dielecric structures can fulfill three important requirements at the same time, when a large number of layers is applied:

i. to act as antireflection coating so to minimize the sample reflectance in the visible [56-59];

ii. to maximize the absorbance in the metallic layers;

iii. to guarantee a low thermal emissivity in the infrared thanks to the metal layers.

Starting from this state of the art, we want to show that it is possible to achieve better results by replacing the metal layers with Titanium Nitride layers (TiN) [50].

In fact TiN is an inexpensive metal alloy with smaller free carrier concentrations than noble metals. It behaves as a diluted metal but with material losses comparable to that of gold, showing an optical penetration depth of about 20-30 nm, in the visible range (see Fig. 1a obtained from the data in ref. [61]). In addition TiN has also good mechanical hardness, high corrosion resistance, low frictional constant, thermodynamic stability, relatively good thermal conductivity $(\approx 20 \mathrm{~W} / \mathrm{m} \mathrm{K})$, low thermal emissivity $(<0.2)$, very high melting temperature of $2930{ }^{\circ} \mathrm{C}$, and a thermal expansion of $9.3 \times 10^{-6} \mathrm{~K}^{-1}$ that that is lower than for gold $\left(14 \times 10^{-6} \mathrm{~K}^{-1}\right)$ and silver $\left(18 \times 10^{-6} \mathrm{~K}^{-1}\right)$. These properties makes it suitable for applications such as thermal radiation engineering and thermophotovoltaics $[62,63]$.
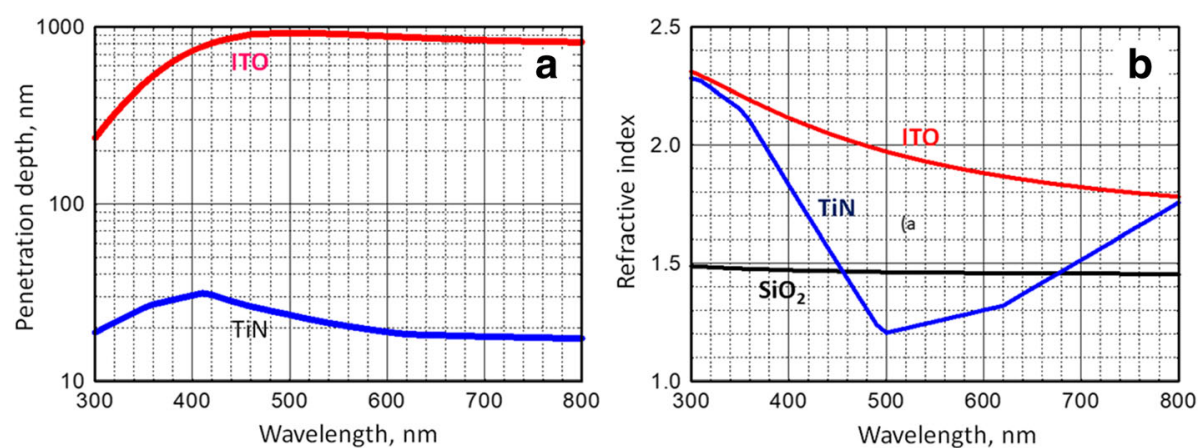

Fig. 1 Penetration depth (a) and refractive index (b) as a function of wavelength in the visible range for ITO, TiN, and $\mathrm{SiO}_{2}$. Penetration depth for silica is not shown because is highly transparent 
In our case the TiN layers are alternated with transparent Indium tin oxide layers (ITO) so to design the ITO/ TiN multilayer structure made of $\mathrm{M}$ layers as shown in Fig. 2. ITO is a transparent conductive oxide, widely used in electronic and optical devices, and compatible with TiN during the deposition process. ITO shows a sufficiently large optical penetration depth of about 700-1000 nm in the visible (see Fig. 1a [64]), with a high refractive index (see Fig. 1b) in the visible, high melting point $\left(>1500{ }^{\circ} \mathrm{C}\right)$, and relatively low thermal emissivity.

An earlier study concerning the combination of these two materials has already shown how the average absorbance can reach $90 \%$ in the visible and for a wide range of incidence angles by using only 7 layers [49]. However we show here that this scheme can be further improved by introducing a silica top layer which acts as an additional antireflection coating (being $n_{\mathrm{SiO} 2} \cong \sqrt{n_{I T O} \cdot n_{\text {air }}}$ ), without any relevant increase of the thermal emissivity, (see Fig. 1b), and by searching the optimal thicknesses of the whole structure with Genetic Algorithms (GAs).

GAs have been introduced in the 60s by John Holland for two purposes [65]: to explain the adaptive processes of natural systems, and to design artificial systems software capable to emulate the mechanisms of natural systems. For many years GAs have been applied to solve both optimization and inverse problems in many different scientific fields: in biology $[66,67]$ in computer science $[68,69]$, in engineering and physics for adaptive filter design [70], in the synthesis of fiber gratings, in thin film metrology [71], in image processing [72], and in metallurgy for the nondestructive testing (NDT) of materials [73-75].

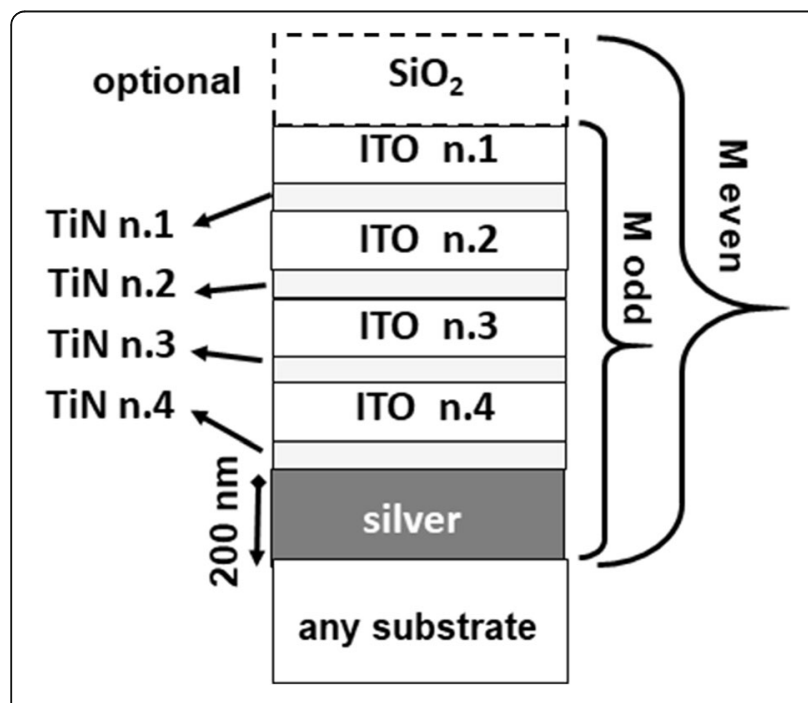

Fig. 2 Scheme of the multilayer structure to be optimized by GAs
Adopting the terminology of the biological sciences, GAs evaluate, process and manipulate a population of chromosomes that represent possible solutions in the research space. In our case the chromosome is a string containing the values of the layer thicknesses of the ITO/TiN structure: for example for $\mathrm{M}$ layers, the chromosome is a string containing $M$ genes $\left\{d_{1}, d_{2}, d_{3}, d_{4}, \ldots, d_{M}\right\}$ where $d_{i}$ is the thickness of the generic $i$-th layer (from top to bottom) (see Fig. 2). For each chromosome, a numerical simulation should be performed to calculate the absorbance of the multilayer by using the transfer-matrix method [76], and to quantify how much the absorbance is close to the ideal case of $100 \%$ through the fitness of the chromosome. Each chromosome belongs to the population of $\mathrm{N}_{\text {pop }}$ individuals. Thanks to the mutual interactions among individuals and to the natural selection mechanisms, the population can evolve and adapt to the environment (research domain), increasing the fitness of all individuals, and eventually finding the best chromosome representing the optimal ITO/TiN multilayer structure.

Once the approach and the methods to optimize the ITO/TiN multilayer structure have been chosen, all the constraints, the requirements and the technical details on the optimization procedure, applied to the design of a heat solar collector, should be also introduced according to the following guidelines:

a) The structure is made of a sequence of ITO and TiN layers. The thickness for each ITO layer should be searched by GAs in the range [ $5 \mathrm{~nm}-100 \mathrm{~nm}$ ], thinner than $\lambda / 2$ so to avoid internal interference effects. Each TiN layer should be searched by GAs in the interval [ $5 \mathrm{~nm}-60 \mathrm{~nm}$ ] so to play with its transparency/opaqueness.

b) A silica top layer can be optionally inserted as additional antireflection coating. Its thickness should be searched by GAs in the range $[5 \mathrm{~nm}-$ $150 \mathrm{~nm}$ ], without causing a relevant change of the IR emissivity.

c) The last $200 \mathrm{~nm}$ thick silver layer absorbs and stops the residual radiation. Therefore the results of the numerical simulations and optimizations are general and independent on the choice of the substrate.

d) The total number of layers $\mathrm{M}$ ranges from 3 to 10 . The silica top layer is inserted only when $M$ is an even number (see Fig. 2). The optimized multilayers found by GAs for different values of $\mathrm{M}$, will be discussed and compared.

e) The objective to be maximized is the absorbance $A_{a v}$ averaged in the visible range from $\lambda_{\min }=400 \mathrm{~nm}$ to $\lambda_{\max }=750 \mathrm{~nm}$, and averaged for a wide range of angles of incidence from $0^{\circ}$ to $\lambda_{\max }=50^{\circ}$, for unpolarised light. It is calculated as follows 


$$
A_{a v}=\frac{\int_{0}^{\theta_{\max }} \int_{\lambda_{\min }}^{\lambda_{\max }}[1-R(\lambda, \theta)] d \lambda d \theta}{\left(\lambda_{\max }-\lambda_{\min }\right) \cdot \theta_{\max }},
$$

where $R(\lambda, \theta)$ is the reflectance for unpolarised light at the wavelength $\lambda$, and at the incident angle $\theta$ and is calculated by using the transfer-matrix method. Note that transmittance is neglected due to the thick silver layer. $A_{a v}$ well represents the figure of merit of the perfect absorber.

A useful related quantity to be calculated is the absorbance for normal incidence $A_{o}$, averaged in the visible again from $\lambda_{\min }=400 \mathrm{~nm}$ to $\lambda_{\max }=750 \mathrm{~nm}$ as follows

$$
A_{o}=\frac{\int_{\lambda_{\min }}^{\lambda_{\max }}[1-R(\lambda, 0)] d \lambda}{\lambda_{\max }-\lambda_{\min }},
$$

When the objective $A_{a v}$ is maximized by GAs, the quantity $A_{o}$ is usually also optimized.

f) The hemispherical total emissivity of the whole structure is also calculated as IR $=1-\mathrm{R}_{\mathrm{IR}}$ in the infrared range from $1 \mu \mathrm{m}$ to $10 \mu \mathrm{m}$, averaged over the solid angle and over the Planck blackbody radiation spectrum for $200{ }^{\circ} \mathrm{C}$ and for $400{ }^{\circ} \mathrm{C}$, which is the typical temperature range for most solar collectors. The hemispherical total emissivity should be kept as small as possible $(<0.2)$ to minimize the radiation losses. The calculation is done by using the literature values for the infrared properties of TiN, ITO, $\mathrm{SiO}_{2}$, and silver [61-64]. g) Many parameters of the GAs has to be set, controlled or adjusted (as will be clear in the next section):

- $\mathrm{M}$ is the number of genes (coincident with the number of layers). It will be selected in the range from 3 to 10 so to design a realistic and sustainable structure;

- $\mathrm{N}_{\text {pop }}$ is the size of the population used for searching the maximum of $\mathrm{A}_{\mathrm{av}}$. It is an even number to be adjusted in the range from 8 to 14;

- $\mathrm{f}$ is the fitness function of each individual and is the quantity to be maximized. It also rules the selection for the reproduction process. In order to enhance the sensitivity we implemented "ad hoc" GAs by using the fitness function $f=1 /\left(1-A_{a v}\right)^{4}$ which is more appropriate to distinguish the difference among highly absorbing structures (with $\mathrm{A}_{\mathrm{av}}$ around unity). This choice allows to reach an optimal solution already after 1000 generations with a run of a few of minutes on a standard PC, giving in general better results with respect to commercial software;

- $\mathrm{P}_{\mathrm{m}}$ is the probability of mutation of each gene in the GAs. It is kept constant to 5\%; This choice is driven by a previous study [30].

\section{Results and discussions}

In this section we show how Genetic Algorithms (GAs) represent a useful tool to find an optimal ITO/TiN multilayer coating for heat solar collectors, giving some examples which demonstrate how the mechanic of the GAs is surprisingly simple and efficient.

According to the methods described in the previous section, the figure of merit of the multilayer coating has

\begin{tabular}{|c|c|c|c|c|c|c|c|c|c|}
\hline \multicolumn{2}{|c|}{$N_{\text {pop }}$ chromosomes $\rightarrow$} & 1 & 2 & 3 & 4 & 5 & 6 & 7 & 8 \\
\hline & $\rightarrow$ & 73.8 & 75.1 & 80.9 & 87.7 & 82 & 89.3 & 66.2 & 77.7 \\
\hline & $\rightarrow$ & 73.9 & 76.6 & 81.1 & 88.8 & 82.8 & 89.2 & 67 & 78.6 \\
\hline Fitnes: & $s\left(\times 10^{-6}\right) \rightarrow$ & 2.2 & 3.3 & 7.8 & 63.2 & 11.4 & 74.3 & 0.8 & 4.7 \\
\hline M genes & Structure & $\mathrm{nm}$ & $\mathrm{nm}$ & $\mathrm{nm}$ & $\mathrm{nm}$ & $\mathrm{nm}$ & $\mathrm{nm}$ & $\mathrm{nm}$ & $\mathrm{nm}$ \\
\hline 1 & $\mathrm{SiO}_{2}$ & 100 & 89 & 52 & 89 & 76 & 49 & 28 & 28 \\
\hline 2 & ITO n.1 & 88 & 7 & 23 & 36 & 55 & 41 & 93 & 18 \\
\hline 3 & TiN n.2 & 54 & 45 & 44 & 25 & 35 & 16 & 58 & 8 \\
\hline 4 & ITO n.2 & 41 & 66 & 99 & 67 & 71 & 29 & 28 & 89 \\
\hline 5 & TiN n.1 & 56 & 38 & 30 & 40 & 43 & 58 & 47 & 23 \\
\hline 6 & Silver & 200 & 200 & 200 & 200 & 200 & 200 & 200 & 200 \\
\hline
\end{tabular}
been identified in the average absorbance $A_{a v}$ or better

Table 1 Initial population processed by the GAs. Number of layer $M=6$. Size of the population $N_{\text {pop }}=8$ 
Table 2 Results after the selection procedure applied to the initial population in Table 1

\begin{tabular}{|c|c|c|c|c|c|c|c|c|c|}
\hline \multicolumn{2}{|c|}{$N_{\text {pop }}$ chromosomes $\rightarrow$} & 1 & 2 & 3 & 4 & 5 & 6 & 7 & 8 \\
\hline & $\rightarrow$ & 89.3 & 89.3 & 87.7 & 89.3 & 89.3 & 87.7 & 80.9 & 87.7 \\
\hline & $A_{a v}$ & 89.2 & 89.2 & 88.8 & 89.2 & 89.2 & 88.8 & 81.1 & 88.8 \\
\hline \multicolumn{2}{|c|}{ Fitness $\left(\times 10^{-6}\right) \rightarrow$} & 74 & 74 & 63 & 74 & 74 & 63 & 8 & 63 \\
\hline M genes & Structure & $\mathrm{nm}$ & $\mathrm{nm}$ & $\mathrm{nm}$ & $\mathrm{nm}$ & $\mathrm{nm}$ & $\mathrm{nm}$ & $\mathrm{nm}$ & $\mathrm{nm}$ \\
\hline 1 & $\mathrm{SiO}_{2}$ & 49 & 49 & 89 & 49 & 49 & 89 & 52 & 89 \\
\hline 2 & ITO n.1 & 41 & 41 & 36 & 41 & 41 & 36 & 23 & 36 \\
\hline 3 & TiN n.1 & 16 & 16 & 25 & 16 & 16 & 25 & 44 & 25 \\
\hline 4 & ITO n.2 & 29 & 29 & 67 & 29 & 29 & 67 & 99 & 67 \\
\hline 5 & TiN n.2 & 58 & 58 & 40 & 58 & 58 & 40 & 30 & 40 \\
\hline 6 & Silver & 200 & 200 & 200 & 200 & 200 & 200 & 200 & 200 \\
\hline
\end{tabular}

in the fitness function $\mathrm{f}=1 /\left(1-\mathrm{A}_{\mathrm{av}}\right)^{4}$ which is more appropriate to enhance the differences among quasi perfect absorbers (when $A_{a v} \approx 1$ ).

As a simple example we start from a ITO/TiN multilayer coating made of $M=6$ layers. We apply GAs to maximize the fitness $f$ (and hence $A_{a v}$ ), finding the optimal thickness of each layer. In this case the generic chromosome has $M=6$ genes and is represented by the string $\left\{d_{1}, d_{2}, d_{3}, d_{4}, d_{5}, d_{6}\right\}$ where $d_{i}$ is the thickness of the generic $\mathrm{i}$-th layer (from top to bottom). Note that according to the scheme in Fig. 2, the silica top layer is included only if $M$ is even. Then for $M=6, d_{1}$ is the thickness of the silica top layer, $d_{2}$, and $d_{3}$ are the thicknesses of the 1st layer of ITO and TiN respectively, $\mathrm{d}_{4}$, and $d_{5}$ are the thicknesses of the 2 nd layer of ITO and
TiN respectively, while $d_{6}=200 \mathrm{~nm}$ is the thickness of the last silver layer that is kept constant (see Table 1).

As said each chromosome identifies a specific individual who belongs to the population of $\mathrm{N}_{\text {pop }}$ individuals. As a result of mutual interactions among individuals, the population can evolve and adapt to the environment, increasing the fitness of all individuals.

Table 1 shows the initial population of $\mathrm{N}_{\text {pop }}=8$ chromosomes. Each chromosome is made of $M=6$ genes (rows from 6 to 11). All genes are randomly chosen within the ranges described in section 2 .

Both absorbances $A_{o}$, and $A_{a v}$ are calculated for each chromosome, and shown in rows 2 , and 3 respectively. The corresponding fitness function $\mathrm{f}=1 /\left(1-\mathrm{A}_{\mathrm{av}}\right)^{4}$ is shown in row 4. Looking at the fitness the best

Table 3 Results after the crossover procedure applied to the population in Table 2

\begin{tabular}{|c|c|c|c|c|c|c|c|c|c|}
\hline \multicolumn{2}{|c|}{$N_{\text {pop }}$ chromosomes $\rightarrow$} & 1 & 2 & 3 & 4 & 5 & 6 & 7 & 8 \\
\hline & $\rightarrow$ & 89.3 & 89.3 & 91.3 & 89.9 & 92.9 & 92.0 & 80.5 & 81.9 \\
\hline & $A_{a v}$ & 89.2 & 89.2 & 91.9 & 89.2 & 93.0 & 93.0 & 82.0 & 83.5 \\
\hline Fitnes & $5\left(\times 10^{-6}\right) \rightarrow$ & 74 & 74 & 237 & 111 & 414 & 358 & 9 & 14 \\
\hline M genes & Structure & $\mathrm{nm}$ & $\mathrm{nm}$ & $\mathrm{nm}$ & $\mathrm{nm}$ & $\mathrm{nm}$ & $\mathrm{nm}$ & $\mathrm{nm}$ & $\mathrm{nm}$ \\
\hline 1 & $\mathrm{SiO}_{2}$ & 49 & 49 & 85 & 80 & 65 & 72 & 85 & 83 \\
\hline 2 & ITO n.1 & 41 & 41 & 39 & 39 & 38 & 37 & 34 & 24 \\
\hline 3 & TiN n.1 & 16 & 16 & 20 & 20 & 19 & 24 & 37 & 34 \\
\hline 4 & ITO n.2 & 29 & 29 & 62 & 43 & 45 & 64 & 80 & 84 \\
\hline 5 & TiN n.2 & 58 & 58 & 48 & 43 & 53 & 46 & 38 & 37 \\
\hline 6 & Silver & 200 & 200 & 200 & 200 & 200 & 200 & 200 & 200 \\
\hline
\end{tabular}


Table 4 Results after the mutation procedure applied to the population in Table 3

\begin{tabular}{|c|c|c|c|c|c|c|c|c|c|}
\hline \multicolumn{2}{|c|}{$N_{\text {pop }}$ Chromosomes $\rightarrow$} & 1 & 2 & 3 & 4 & 5 & 6 & 7 & 8 \\
\hline \multicolumn{2}{|r|}{$A_{0}$} & 89.3 & 91.8 & 91.3 & 82.4 & 92.9 & 87.6 & 80.5 & 78.7 \\
\hline \multicolumn{2}{|r|}{$A_{a v}$} & 89.2 & 91.6 & 91.9 & 83.1 & 93.0 & 88.4 & 82.0 & 79.6 \\
\hline \multicolumn{2}{|c|}{ Fitness $\left(\times 10^{-6}\right) \rightarrow$} & 74 & 203 & 237 & 12 & 414 & 55 & 9 & 6 \\
\hline M genes & Structure & $\mathrm{nm}$ & $\mathrm{nm}$ & $\mathrm{nm}$ & $\mathrm{nm}$ & $\mathrm{nm}$ & $\mathrm{nm}$ & $\mathrm{nm}$ & $\mathrm{nm}$ \\
\hline 1 & $\mathrm{SiO}_{2}$ & 49 & 49 & 85 & 80 & 65 & 98 & 85 & 83 \\
\hline 2 & ITO n.1 & 41 & 41 & 39 & 39 & 38 & 37 & 34 & 24 \\
\hline 3 & TiN n.1 & 16 & 16 & 20 & 38 & 19 & 24 & 37 & 34 \\
\hline 4 & ITO n.2 & 29 & 37 & 62 & 43 & 45 & 64 & 80 & 84 \\
\hline 5 & TiN n.2 & 58 & 58 & 48 & 43 & 53 & 46 & 38 & 37 \\
\hline 6 & Silver & 200 & 200 & 200 & 200 & 200 & 200 & 200 & 200 \\
\hline
\end{tabular}

chromosome of the population is found to be the N.6 (the column is highlighted in black in Table 1).

From Table 1 the best chromosomes are randomly selected for the reproduction with a probability proportional to the fitness value so to give more chances to the best chromosomes respect to the worst ones which risk the extinction. Table 2 shows the new population selected for the reproduction. The best chromosomes can be selected more than once so to transfer the best chromosomic strings to future generations: in fact in Table 2 there are 4 replica of the best chromosome of Table 1 .

After the selection, $\mathrm{N}_{\mathrm{pop}} / 2=4$ couples are formed for reproduction. The reproduction process consists in mixing the genes of each parent couple $(\mathrm{X}, \mathrm{Y})$ so to generate the genes of the two sons $\left(S_{1}, S_{2}\right)$ according to the following rule: $S_{1}=p_{1} X+\left(1-p_{1}\right) Y$, and $S_{2}=p_{2} X+\left(1-p_{2}\right) Y$ where $\mathrm{p}_{1}$ and $\mathrm{p}_{2}$ are weights randomly chosen from 0 to 1. For example in Table 2 the couple of parents N.5 and N.6 generates the couple of sons N.5 and N.6 in Table 3. The first gene of the sons $\left(S_{1}=65, S_{2}=72\right)$ (cells are highlighted in black in Table 3 ) are obtained by mixing the first gene of their parents $(X=49, Y=89)$ (cells are highlighted in black in Table 2) by using the weights $\mathrm{p}_{1}$ $=0.6$ and $\mathrm{p}_{2}=0.42$. This procedure is repeated for all the genes by changing randomly $\mathrm{p}_{1}$ and $\mathrm{p}_{2}$. In this example the fitness of the sons $(414,358)$ is higher than the ones of their parents $(74,63)$. In general after reproduction a new population of children is generated (see Table 3), which statistically has an average fitness better than the previous one (see Table 2), thanks to the selection rules, and to the general assumption that good parents generate good children. In synthesis the new generation tends to adapt more to the environment. Sometimes it happens that after several generations, the individuals become too similar to each other. This unwanted phenomenon may produce an evolutionary stop (epistasis) which inhibits further improvement of the population. To avoid the epistasis, a random mechanism of mutation of the genes should be introduced. Accordingly each single gene may be mutated and substituted by a random value (within the appropriate range seen in section 2) but this may happen only with a small probability (probability of mutation is set to $P_{m}=5 \%$. This value is given by a tradeoff as shown in a previous work [30]. In fact $P_{m} \ll 5 \%$ could cause the epistasis, while $\mathrm{P}_{\mathrm{m}} » 5 \%$ generates random mechanisms for the research of the optimal solution which become dramatically time consuming). This mutation creates a new chromosome, sometimes extremely different from its parents, but anyway useful for the renewal of the population. For example in Table 4, the four genes highlighted in black are mutated

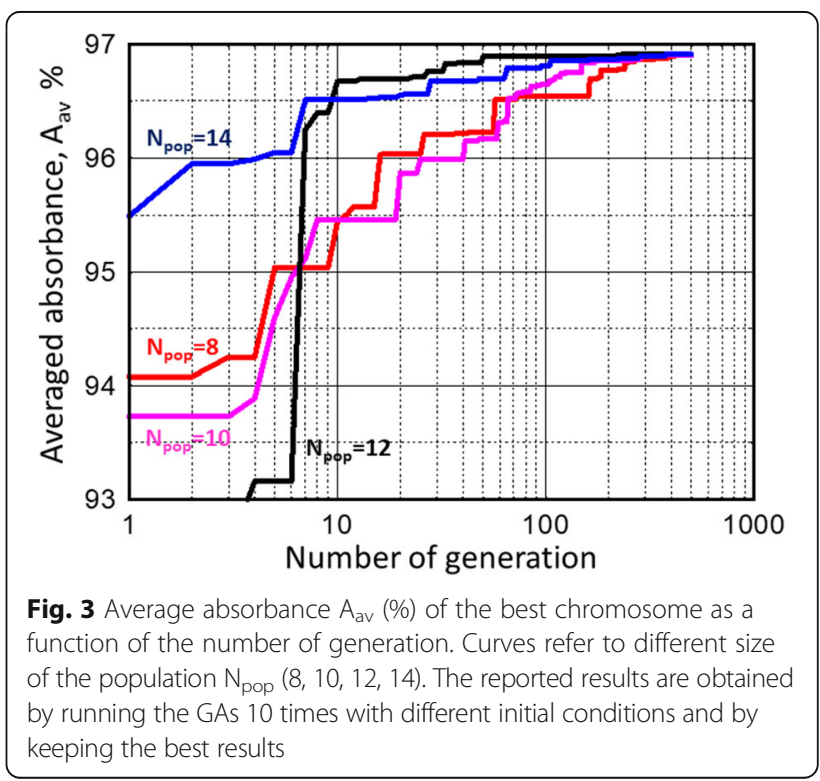




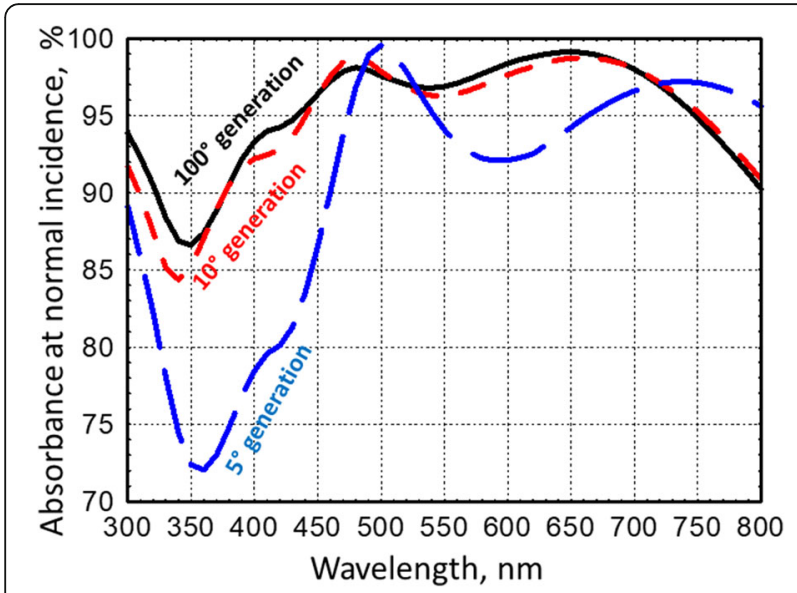

Fig. 4 Absorbance (\%) vs wavelength at normal incidence found by GAs for $M=6$ layers. The curves refer to the results after 5, 10 and 100 generations

with respect to the original genes in Table 3, and by chance the fitness of the chromosome N.2 increases.

Last mechanism of GAs is the elitism, which allows to clone the best chromosome of the previous generation and keep it unchanged for the next generation so to avoid a possible regression of the evolutionary process that might statistically occur: for example chromosome N.1 in Table 4 is the clone of the best chromosome in Table 1.
In synthesis the new generation is formed from the previous one by applying the sequence of the following procedures: selection, reproduction, mutation, and elitism. By iterating these procedures it has been demonstrated that the fitness of the population increases, generation by generation, and that the best chromosome slowly tends to the solution of the optimization problem $[65,30]$.

One fundamental question is now how the evolutionary process can be speeded up so to reduce the computation time needed for finding the optimal structure for the coating of the heat solar collector (which optimizes both $A_{a v}$ and fitness $f$ ). One way is by changing the size of the population $\mathrm{N}_{\text {pop }}$ that is an adjustable parameter of the GAs. The comparison on the performance of GAs for different $\mathrm{N}_{\text {pop }}$ is shown in Fig. 3 where the average absorbance $\mathrm{A}_{\mathrm{av}}$ of the best chromosome is plotted as a function of the number of generations. Each curve refers to a particular value of $\mathrm{N}_{\text {pop }}$ that should be obviously even for reproductive reasons $(8,10,12,14)$. Looking at Fig. 3 there is an optimal value of $\mathrm{N}_{\text {pop }}$ which conjugates the right complexity with the reasonable computational time to find the best solution. In our case the best performances are obtained for $\mathrm{N}_{\mathrm{pop}}=12$, which allows to reach a high value of $A_{a v}$ (about 97\%) already after 100 generations. The corresponding absorbance spectra at normal incidence are shown in Fig. 4 during the iterative process of the GAs, taking the results after 5, 10 and 100 generations. The best chromosome quickly tends to

Table 5 Optimized structures found by GAs for different number of layers M

\begin{tabular}{|c|c|c|c|c|c|c|c|c|}
\hline M Total layers $\rightarrow$ & 3 & 4 & 5 & 6 & 7 & 8 & 9 & 10 \\
\hline$A_{0}[\%] \quad \rightarrow$ & 81.9 & 81.9 & 96.3 & 97.2 & 97.7 & 99.4 & 97.7 & 99.8 \\
\hline $\mathrm{A}_{\mathrm{av}}[\%] \quad \rightarrow$ & 81.4 & 81.4 & 95.8 & 96.9 & 97.1 & 99.1 & 97.1 & 99.5 \\
\hline$\varepsilon_{\mathrm{IR}}[\%]\left(200^{\circ} \mathrm{C}\right) \rightarrow$ & 10.1 & 10.2 & 14.0 & 15.4 & 15.3 & 17.0 & 15.2 & 18.2 \\
\hline$\varepsilon_{\mathrm{IR}}[\%]\left(400^{\circ} \mathrm{C}\right) \rightarrow$ & 11.1 & 11.1 & 15.1 & 16.6 & 16.4 & 18.2 & 16.3 & 19.6 \\
\hline Structure & $\mathrm{nm}$ & $\mathrm{nm}$ & $\mathrm{nm}$ & $\mathrm{nm}$ & $\mathrm{nm}$ & $\mathrm{nm}$ & $\mathrm{nm}$ & $\mathrm{nm}$ \\
\hline $\mathrm{SiO}_{2}$ & & 7 & & 46 & & 58 & & 73 \\
\hline ITO n.1 & 48 & 46 & 51 & 34 & 52 & 29 & 52 & 15 \\
\hline TiN n.1 & 60 & 60 & 20 & 19 & 18 & 14 & 18 & 10 \\
\hline ITO n.2 & & & 54 & 64 & 69 & 67 & 67 & 61 \\
\hline TiN n.2 & & & 60 & 60 & 27 & 21 & 17 & 14 \\
\hline ITO n.3 & & & & & 47 & 48 & 6 & 59 \\
\hline TiN n.3 & & & & & 60 & 59 & 12 & 26 \\
\hline ITO n.4 & & & & & & & 37 & 46 \\
\hline TiN n.4 & & & & & & & 30 & 60 \\
\hline Silver & 200 & 200 & 200 & 200 & 200 & 200 & 200 & 200 \\
\hline
\end{tabular}




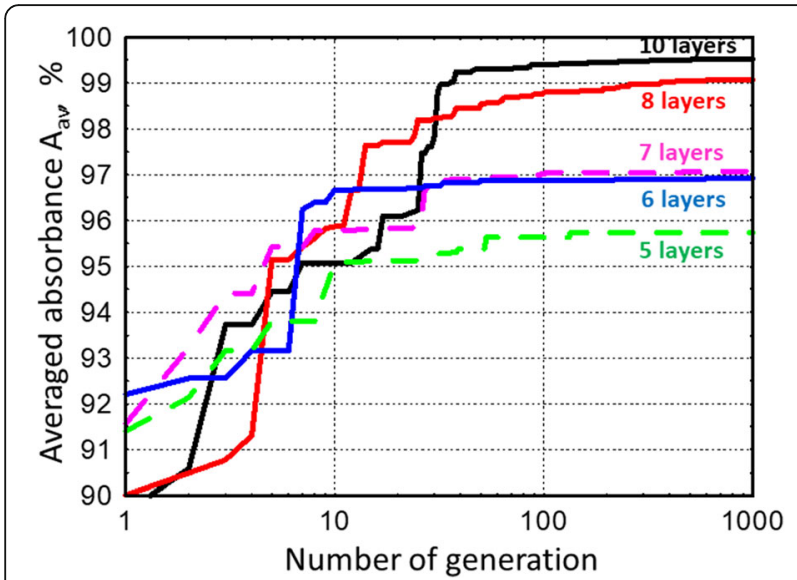

Fig. 5 Average absorbance $A_{a v}(\%)$ of the best chromosome as a function of the number of generation. Curves refer to different number of layers $M(5,6,7,8,10)$. The reported results are obtained by running the GAs 10 times with different initial conditions and by keeping the best results

the optimal string $\{46,34,19,64,60,200\}$ also shown in Table 5 (for $M=6$ ) where the best multilayer structures, calculated by GAs, are summarized for any $M$ number of layers.

In fact GAs have been also applied to optimize the multilayer structures by changing the number of layers $M$ (from $M=3$ to $M=10$, and according to the scheme in Fig. 2). Figure 5 shows the results of the GAs evolution to optimize the average absorbance $\mathrm{A}_{\mathrm{av}}$. The curves refer to several values of $M$, keeping fixed $N_{\text {pop }}=12$. All curves saturate after several hundreds of generations tending asymptotically to the optimized multilayer structures reported in Table 5. In principle the maximum achievable absorbance $\mathrm{A}_{\mathrm{av}}$ should increase with the

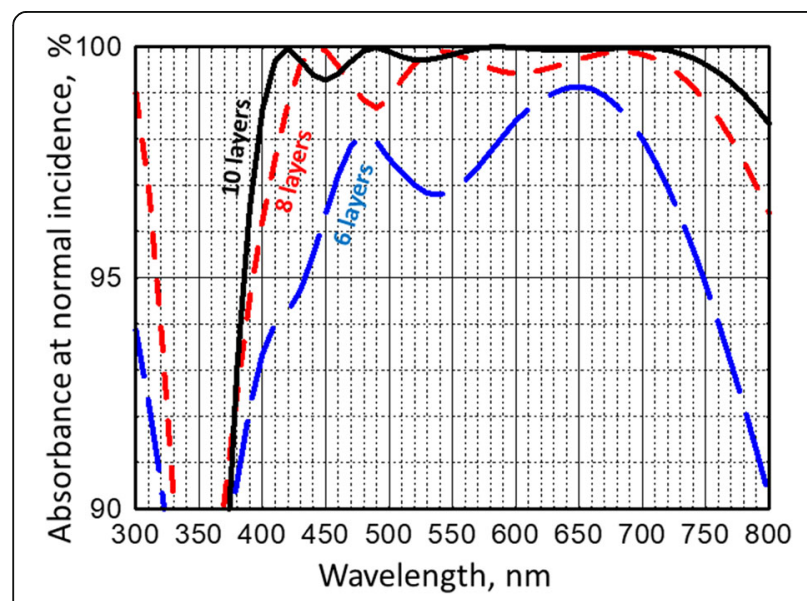

Fig. 6 Absorbance (\%) vs wavelength at normal incidence for three optimized structures. Blue dotted curve is for the structure $\{46,34$, $19,64,60,200\}$ with 6 layers; red dotted curve is for the structure $\{58,29,14,67,21,48,59,200\}$ with 8 layers; black cont. Line is for the structure $\{73,15,10,61,14,59,26,46,60,200\}$ with 10 layers

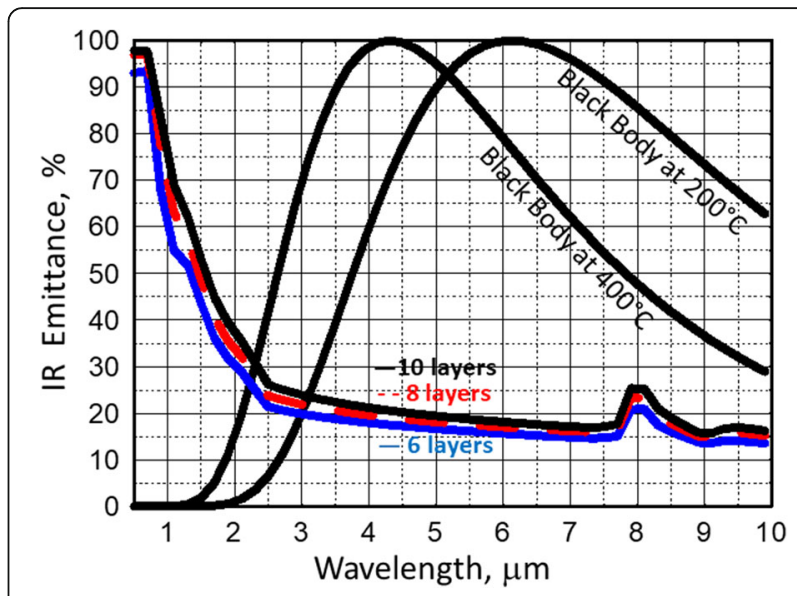

Fig. 7 Infrared emittance (\%) vs wavelength integrated over all the angles and both polarizations for three optimized structures. Blue continuous curve is for the structure $\{46,34,19,64,60,200\}$ with 6 layers; red dotted curve is for the structure $\{58,29,14,67,21,48,59$, $200\}$ with 8 layers; black cont. Line is for the structure $\{73,15,10,61$, $14,59,26,46,60,200\}$ with 10 layers. Radiation curves are also plotted for a blackbody at $200{ }^{\circ} \mathrm{C}$ and at $400{ }^{\circ} \mathrm{C}$. Both curves are normalized to unity

number of layers $M$, but with some exceptions. In fact the best performances are obtained when $M$ is even, when a top silica layer is introduced as an efficient $\lambda / 4$ antireflection coating. This explains why the optimized structure for $M=8$ is better than the one for $M=9$, and why the one for $M=6$ is almost equivalent to the one for $\mathrm{M}=7$.

The absorbance spectra at normal incidence are shown in Fig. 6 only for $M=6,8$, and 10. The corresponding optimized structures are $\{46,34,19,64,60,200\}(M=6)$, $\{58,29,14,67,21,48,59,200\}(M=8)$, and $\{73,15,10$,

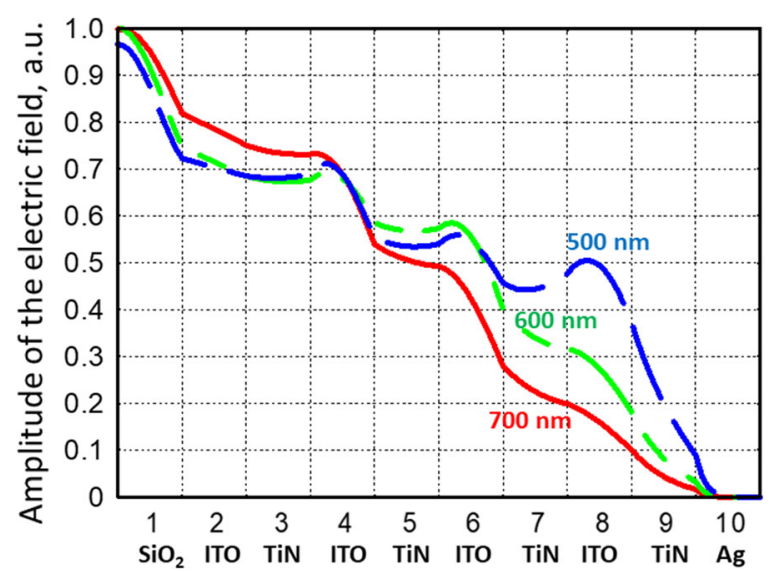

Fig. 8 Amplitude of the internal electric field for the structure $\{73$, $15,10,61,14,59,26,46,60,200\}$ with 10 layers. The curves refers to different wavelengths (500 nm, $600 \mathrm{~nm}, 700 \mathrm{~nm}$ ) for normal incidence. In abscissa the scale of thickness is distorted so to have equally spaced layers 


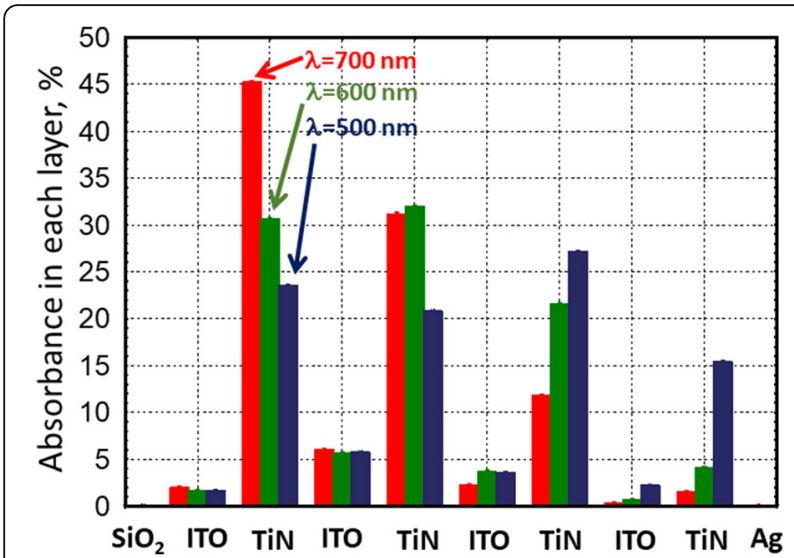

Fig. 9 Specific absorbance in each layer (\%) for the optimized 10 layers structure $\{73,15,10,61,14,59,26,46,60,200\}$ for normal incidence: (blue histogram) $\lambda=500 \mathrm{~nm}$; (green histogram) $\lambda=600 \mathrm{~nm}$; (red histogram) $\lambda=700 \mathrm{~nm}$

$61,14,59,26,46,60,200\}(M=10)$, reaching a very high average absorbance $\mathrm{A}_{\mathrm{av}}$ of $96.9 \%, 99.1 \%$ and $99.5 \%$ respectively.

In order to evaluate the infrared properties of these three optimal structures, we calculate the emissivity spectra from $0.5 \mu \mathrm{m}$ to $10 \mu \mathrm{m}$, integrated for all angles and averaged for both polarizations (see Fig. 7). By a first inspection of Fig. 7 the emissivity drops in the infrared range for all the curves. Finally the hemispherical total emissivity has been also evaluated at two different temperatures $\left(200{ }^{\circ} \mathrm{C}\right.$ and $\left.400{ }^{\circ} \mathrm{C}\right)$ representing the typical thermodynamic range of most solar collectors. In practice the hemispherical total emissivity at $200{ }^{\circ} \mathrm{C}$ and at $400{ }^{\circ} \mathrm{C}$ are calculated by integrating, over the whole spectrum, the emissivity spectra weighted by the black body radiation curves at $200{ }^{\circ} \mathrm{C}$ and at $400{ }^{\circ} \mathrm{C}$ that are shown in Fig. 7 in normalized scale. The values of the hemispherical total emissivity $\varepsilon_{\text {IR }}$ at $200{ }^{\circ} \mathrm{C}$ and at $400{ }^{\circ} \mathrm{C}$ are reported in Table 5 for all the structures. The quantity $\varepsilon_{\text {IR }}$ slightly increases with the number of layers and with temperature, always satisfying the requirement $\varepsilon_{I R}<20 \%$.

To better understand the absorbing properties of the best optimized multilayer obtained with $M=10$, it is useful to study the internal electric field $E$ in the whole structure. Figure 8 shows the amplitude of the E field in each layer at normal incidence. For a better clarity in abscissa the scale of thickness is amplified so to have equally spaced layers. Looking at Fig. 8 an efficient mechanism of distributed absorption is seen at the two wavelengths of $600 \mathrm{~nm}$ and $700 \mathrm{~nm}$. Light penetrates without relevant reflection through the structures, being absorbed by the TiN layers and eventually by the silver layer. Conversely the absorption mechanism at $500 \mathrm{~nm}$ is related to the light trapping and quasi-resonant absorption [77].

The evidence of the different absorption mechanisms is given by showing the absorbance in each layer along the thin film stack. It is calculated from the difference between the incoming and outgoing Poynting vectors in each layer. Figure 9 show the distribution of the absorbance for the optimized structure with $M=10\{73,15,10$, $61,14,59,26,46,60,200\}$, for normal incidence, at $\lambda=$ $500 \mathrm{~nm}$ (blue histogram), $\lambda=600 \mathrm{~nm}$ (green histogram), and $\lambda=700 \mathrm{~nm}$ (red histogram). The absorption occurs prevalently in the TiN layers, but with some differences: at $700 \mathrm{~nm}$ the light is absorbed in the first TiN layers, while at $500 \mathrm{~nm}$, the absorption is enhanced in the internal layers due to a resonance process.

To better analyse the absorbing properties of the structures the contour plots in Fig. 10 represent a useful tool to put together both the wavelength and angular behaviour of the absorbance for two optimized multilayers: for $M=6$ layers (Fig. 10a) and for $M=10$ layers (Fig. 10b). By comparing the two plots the optimized structure with $M=10$ layers obviously shows the best performances in the dotted rectangular area for angles in the range $\left[0^{\circ}-50^{\circ}\right]$ and for wavelengths in the visible range [400 nm - $750 \mathrm{~nm}$ ] which guarantees a huge average

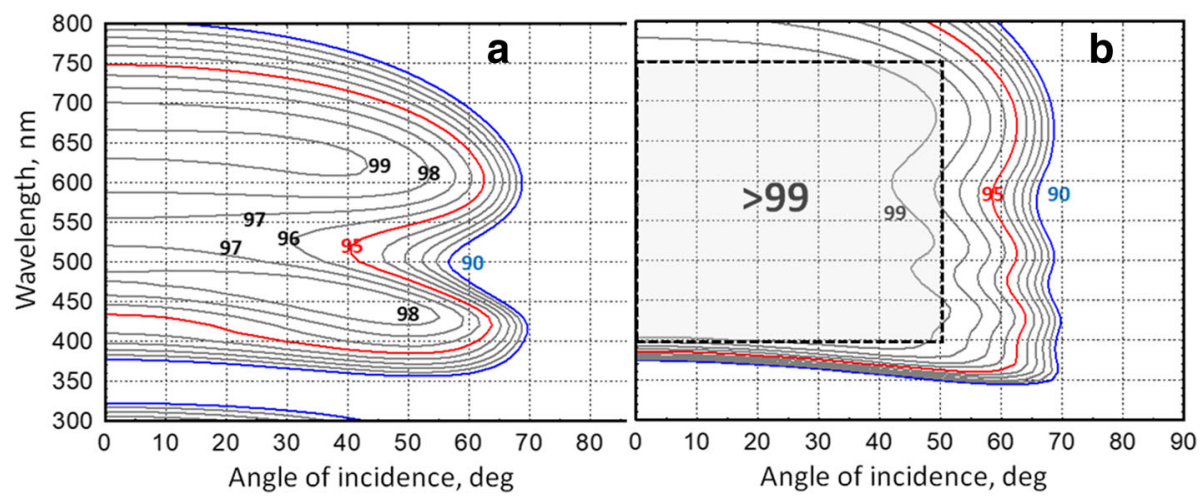

Fig. 10 Contour plot for the absorbance as a function of the angle of incidence ( $x$ axis), and as a function of the wavelengths ( $y$ axis): (a) plot for the optimized 6 layers structure $\{46,34,19,64,60,200\}$; (b) plot for the optimized 10 layers structure $\{73,15,10,61,14,59,26,46,60,200\}$ 
absorbance of $99.5 \%$ inside the rectangle. However the results for $\mathrm{M}=6$ layers are also interesting, showing $\mathrm{A}_{\mathrm{av}} \approx 97 \%$ but by using a smaller number of layers. In other words the performance increases with the number of layers (see Table 5), and it is understandable that the best sustainable practical solution should be always given by a trade off between the required performance and the structure complexity. It is also worth noting that all structures show a low hemispherical total emissivity till $400{ }^{\circ} \mathrm{C}(<20 \%)$, fulfilling the requirement for the efficient solar heat collectors.

\section{Conclusions}

The design of quasi perfect absorbers based on ITO/TiN multilayered structures is here discussed. The practical purpose is to find new optimized coatings for solar thermal collectors with the highest achievable absorbance in the visible range from $400 \mathrm{~nm}$ to $750 \mathrm{~nm}$, working for a wide range of angles of incidence from $0^{\circ}$ to $50^{\circ}$, for both polarizations, and with a low hemispherical total emissivity, so to minimize the radiative losses. Genetic Algorithms are introduced and adjusted for searching the optimal thicknesses for several ITO/TiN multilayered structures with 6, 8, 10 layers reaching a very high average absorbance of $97 \%, 99 \%$ and $99.5 \%$ respectively and a low hemispherical total emissivity $(<20 \%)$ from $200{ }^{\circ} \mathrm{C}$ till $400{ }^{\circ} \mathrm{C}$. The proposed multilayer structures use materials with high thermal stability, and high melting temperature, can be fabricated with simple thin film deposition techniques, appearing to have very promising applications in solar thermal energy harvesting.

\section{Abbreviations \\ $A_{a v}$ : Absorbance averaged in the wavelength range $400 \mathrm{~nm}-750 \mathrm{~nm}$, and in the angular range from $0^{\circ}$ to $50^{\circ} ; A_{\circ}$ : Absorbance for normal incidence averaged in the wavelength range $400 \mathrm{~nm}-750 \mathrm{~nm}$; $\varepsilon_{\mathbb{R}}$ : hemispherical total emissivity; f: Fitness function of the Genetic Algorithms; GAs: Genetic Algorithms; ITO: Indium Tin Oxide; M: Number of layers of the structure; $\mathrm{N}_{\text {pop }}$ : Size of the population of the Genetic Algorithms; $\mathrm{P}_{\mathrm{m}}$ : Probability of mutation; $\mathrm{SiO}_{2}$ : silica; TiN: Titanium Nitride}

\section{Acknowledgements}

This author is indebted with Mario Bertolotti and Joseph Haus for useful discussions.

\section{Funding}

This work has been done in the framework of the project "optical metamaterial" funded by Sapienza University of Rome, and "Scherma" co-financed by Italian Ministry of Defence.

\section{Availability of data and materials}

The numerical results and data can be reproduced by applying the optical method in Ref. [71], by using the Genetic Algorithms described in Ref. [60], and by taking the optical properties of the materials found in Refs. [47, 61-64]. No additional supporting information or data are necessary.

\section{Author's contributions}

The author read and approved the final manuscript.

\section{Author's information}

Roberto Li Voti is associate professor in Applied Physics at Sapienza University of Rome, Italy. He is author of more than 150 publications in the field of optics, photohermal and photoacoustic techniques for nondestructive testing of materials.

\section{Competing interests}

The author declares that he has no competing interests.

\section{Publisher's Note}

Springer Nature remains neutral with regard to jurisdictional claims in published maps and institutional affiliations.

Received: 18 November 2017 Accepted: 28 March 2018

Published online: 10 April 2018

\section{References}

1. Siegel, R., Howell, J.R.: Thermal Radiation Heat Transfer, vol. 1, 4th edn, p. 7. Taylor \& Francis (2002) ISBN 1-56032-839-8.

2. Nam, Y., Xiang Yeng, Y., Lenert, A., Bermel, P., Celanovic, I., Soljačić, M., Wang, E.N.: Solar thermophotovoltaic energy conversion systems with two-dimensional tantalum photonic crystal absorbers and emitters. Sol. Energy Mater. Sol. Cells. 122, 287-296 (2014)

3. Mizuno, K., et al.: A black body absorber from vertically aligned singlewalled carbon nanotubes. Proc Natl Acad Sci. 106, 6044-6077 (2009). https://doi.org/10.1073/pnas.0900155106

4. Tune, D.D., Flavel, B.S., Krupke, R., Shapter, J.G.: Solar Cells: Carbon Nanotube-Silicon Solar Cells. Advanced Energy Materials. 2, 1043-1055 (2012). https://doi.org/10.1002/aenm.201290045

5. Leahu, G., Li Voti, R., Larciprete, M.C., Sibilia, C., Bertolotti, M., Nefedov, I., Anoshkin, I.V.: Thermal Characterization of Carbon Nanotubes by Photothermal Techniques. Int. J. Thermophys. 36, 1349-1357 (2015)

6. Cao, T., Wei, C.W., Simpson, R.E., Zhang, L., Cryan, M.J.: Broadband Polarization-Independent Perfect Absorber Using a Phase-Change Metamaterial at Visible Frequencies. Sci. Rep. 4, 3955 (2014)

7. Wang, W., Wu, S., Reinhardt, K., Lu, Y., Chen, S.: Broadband Light Absorption Enhancement in Thin-Film Silicon Solar Cells. Nano Lett. 10, 2012 (2010)

8. Aydin, K., Ferry, V.E., Briggs, R.M., Atwater, H.A.: Broadband polarizationindependent resonant light absorption using ultrathin plasmonic super absorbers. Nat. Commun. 2, 517 (2011)

9. Teperik, T.V., De Abajo, F.G., Borisov, A., Abdelsalam, M., Bartlett, P., Sugawara, Y., Baumberg, J.: Omnidirectional absorption in nanostructured metal surfaces. Nat. Photonics. 2, 299 (2008)

10. Cheng, C.W., Abbas, M.N., Chiu, C.W., Lai, K.T., Shih, M.H., Chang, Y.C.: Wideangle polarization independent infrared broadband absorbers based on metallic multi-sized disk arrays. Opt. Express. 20, 10376 (2012)

11. Centini, M., Benedetti, A., Larciprete, M.C., Belardini, A., Li Voti, R., Bertolotti, M., Sibilia, C.: Midinfrared thermal emission properties of finite arrays of gold dipole nanoantennas. Phys. Rev. B. 92, 205411 (2015)

12. Li Voti, R., Leahu, G., Larciprete, M.C., Sibilia, C., Bertolotti, M., Nefedov, I., Anoshkin, I.V.: Photoacoustic Characterization of Randomly Oriented Silver Nanowire Films. Int. J. Thermophys. 36, 1342-1348 (2015)

13. Belardini, A., Pannone, F., Leahu, G., Larciprete, M.C., Centini, M., Sibilia, C., Martella, C., Giordano, M., Chiappe, D., Buatier de Mongeot, F.: Evidence of anomalous refraction of self-assembled curved gold nanowires. Appl. Phys. Lett. 100(25), 251109 (2012)

14. Laroche, M., Carminati, R., Greffet, J.J.: Coherent Thermal Antenna Using a Photonic Crystal Slab. Phys. Rev. Lett. 96, 123903 (2006)

15. Celanovic, I., Perreault, D., Kassakian: Resonant-cavity enhanced thermal emission. J. Phys. Rev. B. 72, 075127 (2005)

16. Hu, C.G., Liu, L.Y., Chen, X.N., Luo, X.G.: Mixed plasmons coupling for expanding the bandwidth of near-perfect absorption at visible frequencies. Opt. Express. 17, 16745 (2009)

17. Leahu, G., Voti, R.L., Sibilia, C., Bertolotti, M., Golubev, V., Kurdyukov, D.A.: Study of thermal and optical properties of $\mathrm{SiO}_{2} / \mathrm{GaN}$ opals by photothermal deflection technique. Opt. Quant. Electron. 39, 305-310 (2007)

18. Kats, M.A., Blanchard, R., Zhang, S., Genevet, P., Ko, C., Ramanathan, S., Capasso, F.: Vanadium Dioxide as a Natural Disordered Metamaterial: Perfect Thermal Emission and Large Broadband Negative Differential Thermal Emittance. Physical Review X. 3, 041004 (2013) 
19. Paonea, M., Geiger, R., Sanjines, A., Schüler: Thermal solar collector with $\mathrm{VO}_{2}$ absorber coating and thermochromic glazing - Temperature matching and triggering. Sol. Energy. 110, 151-159 (2014)

20. Leahu, G., Li Voti, R., Sibilia, C., Bertolotti, M.: Anomalous optical switching and thermal hysteresis during semiconductor-metal phase transition of VO2 films on Si substrate. Appl. Phys. Lett. 103, 231114 (2013)

21. Voti, R.L., Larciprete, M.C., Leahu, G., Sibilia, C., Bertolotti, M.: Optimization of thermochromic VO 2 based structures with tunable thermal emissivity. J. Appl. Phys. 112, 034305 (2012)

22. Mercuri, F., Zammit, U., Scudieri, F., Marinelli, M.: Thermal and optical study of the kinetics of the nematic-isotropic transition in octylcyanobiphenyl. Phys. Rev. E. 68, 041708 (2003)

23. Zammit, U., Marinelli, M., Mercuri, F., Paoloni, S.: Effect of Confinement and Strain on the Specific Heat and Latent Heat over the Nematic-Isotropic Phase Transition of 8CB Liquid Crystal. J. Phys. Chem. B. 113, 14315-14322 (2009)

24. Wang, B., Koschny, T., Soukoulis, C.M.: Wide-angle and polarization-independent chiral metamaterial absorber. Phys. Rev. B. 80(033108), (2009)

25. Plum, E., Zheludev, N.I.: Chiral mirrors. Appl. Phys. Lett. 106(221901), (2015)

26. Belardini, A., Centini, M., Leahu, G., Hooper, D.C., Li Voti, R., Fazio, E., Haus, J.W., Sibilia, C.: Chiral light intrinsically couples to extrinsic/pseudo-chiral metasurfaces made of tilted gold nanowires. Sci. Rep. 6, 31796 (2016)

27. Benedetti, A., Alam, B., Esposito, M., Tasco, V., Leahu, G., Belardini, A., Li Voti, R. Passaseo, A., Sibilia, C.: Precise detection of circular dichroism in a cluster of nano-helices by photoacoustic measurements. Sci. Rep. 7, 5257 (2017)

28. Wu, J., Zhou, C.Z., Cao, H.C., Hu, A.D.: Polarization-dependent and -independent spectrum selective absorption based on a metallic grating structure. Opt. Comm. 309, 57 (2013)

29. Chen, H.-T., Zhou, J., O'Hara, J.F., Chen, F., Azad, A.K., Taylor, A.J.: Antireflection Coating Using Metamaterials and Identification of Its Mechanism. Phys. Rev. Lett. 105(073901), (2010)

30. Li Voti, R.: Optimization of transparent metal structures by genetic algorithms. Romanian Reports in Physics. 64, 446-466 (2012)

31. Glorieux, C., Thoen, J.: Thermal depth profile reconstruction by neural network recognition of the photothermal frequency spectrum. J. Appl. Phys. $80,6510(1996)$

32. Glorieux, R., Thoen, L.V.J., Bertolotti, M., Sibilia, C.: Depth profiling of thermally inhomogeneous materials by neural network recognition of photothermal time domain data. J. Appl. Phys. 85, 7059-7063 (1999)

33. Glorieux, C., Li Voti, R., Thoen, J., Bertolotti, M., Sibilia, C.: Photothermal depth profiling: Analysis of reconstruction errors. Inverse Problems. 15 1149-1163 (1999)

34. Tomoda, M., Li Voti, R., Matsuda, O., Wright, O.B.: Tomographic reconstruction of picosecond acoustic strain propagation. Appl. Phys. Lett. 90, $041114(2007)$

35. Krapez, J.C., Li Voti, R.: Effusivity Depth Profiling from Pulsed Radiometry Data: Comparison of Different Reconstruction Algorithms. Anal. Sci. 17, s417-s418 (2001). https://doi.org/10.14891/analscisp.17icpp.0.s417.0

36. Li Voti, R., Leahu, G.L., Gaetani, S., Sibilia, C., Violante, V., Castagna, E., Bertolotti, M.: Light scattering from a rough metal surface: Theory and experiment. J. Opt. Soc. Am. B. 26, 1585-1593 (2009)

37. Larciprete, M.C., Belardini, A., Voti, R.L., Sibilia, C.: Pre-fractal multilayer structure for polarizationinsensitive temporally and spatially coherent thermal emitter. Opt. Express. 21, A576-A584 (2013)

38. Larciprete, M.C., Centini, M., Voti, R.L., Bertolotti, M., Sibilia, C.: Polarization insensitive infrared absorbing behaviour of one-dimensional multilayer stack: A fractal approach. Opt. Express. 22, A1547-A1552 (2014)

39. Melnikov, A., Mandelis, A., Tolev, J., Chen, P., Huq, S.: Infrared lock-in carrierography (photocarrier radiometric imaging) of Si solar cells. J. Appl. Phys. 107, $114513(2010)$

40. Sun, Q.M., Melnikov, A., Mandelis, A.: Quantitative Carrier Density Wave Imaging in Silicon Solar Cells Using Photocarrier Radiometry and Lock-in Carrierography. Int. J. Thermophys. 37, 45 (2016)

41. Matsuda, O., Larciprete, M.C., Li Voti, R., Wright, O.B.: Fundamentals of picosecond laser ultrasonics. Ultrasonics. 56, 3-20 (2015)

42. Dehoux, T., Wright, O.B., Voti, R.L.: Picosecond time scale imaging of mechanical contacts. Ultrasonics. 50, 197-201 (2010)

43. Tomoda, M., Wright, O.B., Li Voti, R.: Nanoscale thermoelastic probing of megahertz thermal diffusion. Appl. Phys. Lett. 91, 071911 (2007)

44. Zammit, U., Mercuri, F., Paoloni, S., Marinelli, M., Pizzoferrato, R.: Simultaneous absolute measurements of the thermal diffusivity and the thermal effusivity in solids and liquids using photopyroelectric calorimetry. J. Appl. Phys. 117, 105104 (2015)

45. Zammit, U., Paoloni, S., Mercuri, F., Marinelli, M., Scudieri, F.: Self consistently calibrated photopyroelectric calorimeter for the high resolution simultaneous absolute measurement of the specific heat and of the thermal conductivity. AlP Advances. 2, 012135 (2012)

46. Naik, G.V., Kim, J., Boltasseva, A.: Oxides and nitrides as alternative plasmonic materials in the optical range. Opt. Mater. Express. 1, 1090 (2011)

47. Naik, G.V., Schroeder, J.L., Ni, X., Kildishev, A.V., Sands, T.D., Boltasseva, A.: Titanium nitride as a plasmonic material for visible and near-infrared wavelengths. Opt. Mater. Express. 2, 478 (2012)

48. Naik, G.V., Shalaev, V.M., Boltasseva, A.: Alternative Plasmonic Materials: Beyond Gold and Silver. Adv. Mater. 25, 3264 (2013)

49. Wang, J., Yin, C., Zhu, M., Sun, J., Yi, K., Shao, J.: Wide angle and broadband perfect absorber with compact multilayer structures. Mod. Phys. Lett. B. 31, 1750136 (2017)

50. Venugopal, N., Gerasimov, V.S., Ershov, A.E., Karpov, S.V., Polyutov, S.P.: Titanium nitride as light trapping plasmonic material in silicon solar cell. Opt. Mater. 72, 397-402 (2017)

51. Yu, H., Tan, T., Wu, W., Tian, C., An, Y., Sun, F.: Thermal stability of titanium nitride coatings prepared by the mixing technology with laser and plasma. Curr. Appl. Phys. 12, 152-154 (2012)

52. Liu, Y., Mandelis, A., Choy, M., Wang, C., Lee, S.: Remote quantitative temperature and thickness measurements of plasma-deposited titanium nitride thin coatings on steel using a laser interferometric thermoreflectance optical thermometer. Rev. Sci. Instrum. 76, 084902 (2005)

53. Abb, M., Sepu'lveda, B., Chong, M.H., Muskens, O.L.: Transparent conducting oxides for active hybrid metamaterial devices. J. Opt. 14, 114007-114001 (2012)

54. Rajak, S., Ray, M.: Comparative study of plasmonic resonance in transparent conducting oxides: ITO and AZO. J. Opt. 43, 231 (2014)

55. You, J.-B., Lee, W.-J., Won, D., Yu, K.: Multiband perfect absorbers using metal-dielectric films with optically dense medium for angle and polarization insensitive operation. OPTICS EXPRESS. 22, 8339 (2014)

56. Kuo, C.-C.: International Scholarly and Scientific Research \& Innovation. 7, 570-572 (2013)

57. Scalora, M., Bloemer, M.J., Pethel, A.S., Dowling, J.P., Bowden, C.M., Manka, A.S.: Transparent, metallo-dielectric, one-dimensional, photonic band-gap structures. J. Appl. Phys. 83, 2377 (1998)

58. Sarto, M.S., Li Voti, R., Sarto, F., Larciprete, M.C.: Nanolayered lightweight flexible shields with multidirectional optical transparency. IEEE Transactions on Electromagnetic Compatibility. 47, 602-611 (2005)

59. Li Voti, R., Larciprete, M.C., Leahu, G., Sibilia, C., Bertolotti, M.: Optical response of multilayer thermochromic VO2-based structures. Journal of Nanophotonics. 6, 061601 (2012)

60. Cesarini, G., Leahu, G., Grilli, M.L., Sytchkova, A., Sibilia, C., Voti, R.L.: Optical and photoacoustic investigation of AZO/Ag/AZO transparent conductive coating for solar cells. Phys. Status Solidi C. 13, 998-1001 (2016)

61. Pflüger, J., Fink, J., Weber, W., Bohnen, K.P., Crecelius, G.: Dielectric properties of TiCx, TiNx, VCx, and VNx from 1.5 to $40 \mathrm{eV}$ determined by electronenergy-loss spectroscopy. Phys. Rev. B. 30, 1155-1163 (1984)

62. Yuste, M., Escobar Galindo, R., Sánchez, O., Cano, D., Casasola, R., Albella, J.M. Correlation between structure and optical properties in low emissivity coatings for solar thermal collectors. Thin Solid Films. 518, 5720 (2010)

63. Briggs, J.A., Naik, G.V., Yang, Z., Petach, T.A., Sahasrabuddhe, K., Gordon, D.G., Melosh, N.A., Dionne, J.A.: Temperature-dependent optical properties of titanium nitride. Appl. Phys. Lett. 110, 101901 (2017)

64. Palik, E.D.: Handbook of Optical Constants of Solids. Academic Press, New York (1985)

65. Holland, J.H.: Outline for a Logical Theory of Adaptive Systems. J. Assoc. Comput. Mach. 3, 297-314 (1962)

66. Rosenberg, R.S.: Simulation of genetic populations with biochemical properties : I. The model. Math. Biosci. 7, 223-257 (1970)

67. Rosenberg, R.S.: Simulation of genetic populations with biochemical properties: II. Selection of crossover probabilities. Math. Biosci. 8, 1-37 (1970)

68. David Edward Goldberg, Genetic Algorithms in Search, Optimization, and Machine Learning, Publisher: Addison-Wesley Professional; 1 edition (Jan. 1 1989) ISBN-10: 0201157675, ISBN-13: 978-0201157673.

69. Etter, D.M., Hicks, M.J., Cho, K.H.: Recursive adaptive filter design using an adaptive genetic algorithm. IEEE Int Conference Acoustics Speech Signal Proc. 2, 635-638 (1982) 
70. Goldberg D.E., "Computer-aided gas pipeline operation using genetic algorithms and rule learning", Dissertation Abstracts International 44 (10) 3174b (1983). DOI (doi:https://doi.org/10.1234/12345678), or a PubMed ID (pmid:12345678).

71. Skaar, J., Risvik, K.M.: A Genetic Algorithm for the Inverse Problem in Synthesis of Fiber Gratings. J. Lightwave Technol. 16, 1928-1932 (1998)

72. Lienert, B.R., Porter, J.N., Sharma, S.K.: Repetitive genetic inversion of optical extinction data. Appl. Opt. 40, 3476-3482 (2001)

73. Li Voti, R.: Inverse problems by Genetic Algorithms: application to the photothermal depth profiling. In: Maldague, X., ASNT (eds.) IV International Workshop - Advances in Signal Processing for Nondestructive Evaluation of Materials, vol. 6, pp. 31-41. Published by The American Society Nondestructive Testing Inc. (2002) ISBN: 1-57117-091-X

74. Li Voti, R., Melchiorri, C., Sibilia, C., Bertolotti, M.: Use of the Genetic Algorithms in the Photothermal Depth Profiling. Anal. Sci. 17, s410-s413 (2001). https://doi.org/10.14891/analscisp.17icpp.0.s410.0

75. Li Voti, R., Sibilia, C., Bertolotti, M.: Photothermal depth profiling by thermal wave backscattering and genetic algorithms. Int. J. Thermophys. 26, 1833-1848 (2005)

76. Born, M., Wolf, E.: Principles of optics: electromagnetic theory of propagation, interference and diffraction of light. Pergamon Press, Oxford (1964)

77. Leahu, G., Petronijevic, E., Belardini, A., Centini, M., Li Voti, R., Hakkarainen, T., Koivusalo, E., Guina, M., Sibilia, C.: Photo-acoustic spectroscopy revealing resonant absorption of self-assembled GaAs-based nanowires. Sci. Rep. 7, $2833(2017)$

\section{Submit your manuscript to a SpringerOpen ${ }^{\circ}$ journal and benefit from:}

- Convenient online submission

- Rigorous peer review

- Open access: articles freely available online

- High visibility within the field

- Retaining the copyright to your article

Submit your next manuscript at $\gg$ springeropen.com 